\title{
The missing link in gravitational-wave astronomy
}

\section{A summary of discoveries waiting in the decihertz range}

\author{
Manuel Arca Sedda ${ }^{1}$. Christopher P. L. Berry ${ }^{2,3}$ (D) Karan Jani ${ }^{4}$.
}

Pau Amaro-Seoane ${ }^{5,6,7,8}$. Pierre Auclair ${ }^{9}$. Jonathon Baird ${ }^{10}$. Tessa Baker $^{11}$.

Emanuele Berti $^{12}$. Katelyn Breivik ${ }^{13}$. Chiara Caprini ${ }^{9}$. Xian Chen ${ }^{6,14}$.

Daniela Doneva ${ }^{15}$. Jose M. Ezquiaga ${ }^{16}$ - K. E. Saavik Ford ${ }^{17,18}$.

Michael L. Katz ${ }^{2}$. Shimon Kolkowitz ${ }^{19}$ • Barry McKernan 17,18 . Guido Mueller ${ }^{20}$. Germano Nardini $^{21}$ - Igor Pikovski ${ }^{22,23}$. Surjeet Rajendran ${ }^{12}$.

Alberto Sesana ${ }^{24}$. Lijing Shao ${ }^{6,25}$. Nicola Tamanini ${ }^{26}$. Niels Warburton ${ }^{27}$. Helvi Witek ${ }^{28} \cdot$ Kaze Wong $^{12} \cdot$ Michael Zevin $^{2}$

Received: 3 August 2020 / Accepted: 9 February 2021 / Published online: 29 April 2021

(C) The Author(s) 2021

\begin{abstract}
Since 2015 the gravitational-wave observations of LIGO and Virgo have transformed our understanding of compact-object binaries. In the years to come, ground-based gravitational-wave observatories such as LIGO, Virgo, and their successors will increase in sensitivity, discovering thousands of stellar-mass binaries. In the 2030s, the space-based LISA will provide gravitational-wave observations of massive black holes binaries. Between the $\sim 10-10^{3} \mathrm{~Hz}$ band of ground-based observatories and the $\sim 10^{-4}-10^{-1} \mathrm{~Hz}$ band of LISA lies the uncharted decihertz gravitational-wave band. We propose a Decihertz Observatory to study this frequency range, and to complement observations made by other detectors. Decihertz observatories are well suited to observation of intermediate-mass $\left(\sim 10^{2}-10^{4} M_{\odot}\right)$ black holes; they will be able to detect stellar-mass binaries days to years before they merge, providing early warning of nearby binary neutron star mergers and measurements of the eccentricity of binary black holes, and they will enable new tests of general relativity and the Standard Model of particle physics. Here we summarise how a Decihertz Observatory could provide unique insights into how black holes form and evolve across cosmic time, improve prospects for both multimessenger astronomy and multiband gravitational-wave astronomy, and enable new probes of gravity, particle physics and cosmology.
\end{abstract}

Christopher P. L. Berry

christopher.berry@northwestern.edu 
Keywords Gravitational waves · Decihertz observatories ·

Multiband gravitational-wave astronomy · Multimessenger astronomy ·

Space-based detectors · Black holes $\cdot$ Neutron stars $\cdot$ White dwarfs ·

Stochastic backgrounds · Binary evolution · Intermediate-mass black holes .

Tests of general relativity · Voyage 2050

\section{The gravitational-wave spectrum}

When new frequency ranges of the electromagnetic spectrum became open to astronomy, our understanding of the Universe expanded as we gained fresh insights and discovered new phenomena [1]. Equivalent breakthroughs are awaiting gravitationalwave (GW) astronomy [2,3]. Here, we summarise the scientific potential of exploring the $\sim 0.01-1 \mathrm{~Hz} G W$ spectrum.

The first observation of a GW signal was made in 2015 by the Laser Interferometer Gravitational-Wave Observatory (LIGO) [3]. Ground-based detectors such as LIGO [4], Virgo [5], and KAGRA [6] observe over a frequency spectrum $\sim 10$ $10^{3} \mathrm{~Hz}$. This is well tailored to the detection of merging stellar-mass black hole (BH) and neutron star (NS) binaries [7]. Next-generation ground-based detectors, like Cosmic Explorer [8] or the Einstein Telescope [9, 10] may observe down to a few hertz. Only a small part of the GW spectrum can thus be observed by ground-based detectors, and extending to lower frequencies requires space-based observatories.

Lower frequency GW signals originate from coalescences of more massive binaries, and stellar-mass binaries earlier in their inspirals. Due for launch in 2034, the Laser Interferometer Space Antenna (LISA) will observe across frequencies $\sim 10^{-4}$ $10^{-1} \mathrm{~Hz}$ [11], optimal for mergers of binaries with $\sim 10^{6} M_{\odot}$ massive BHs [12-14]. LISA will be able to observe nearby stellar-mass binary BHs (BBHs) years-days prior to merger [15], when they could be observed by ground-based detectors. Multiband observations of BBHs would provide improved measurements of source properties [16-18], new constraints on their formation channels [19, 20], and enable precision tests of general relativity (GR) $[16,21]$.

Pulsar timing arrays are sensitive to even lower frequency GWs of $\sim 10^{-9}$ $10^{-7} \mathrm{~Hz}$ [22], permitting observation of $\sim 10^{9} M_{\odot}$ supermassive BHs [23]. Combining LISA and pulsar timing observations will produce new insights into the evolution of (super)massive BHs [24, 25].

The case for extending the accessible GW spectrum with an observatory that can observe in the $\sim 0.01-1 \mathrm{~Hz}$ decihertz range is explained in [26], based upon a White Paper that we submitted in response to ESA's Voyage 2050 call, and here we summarise the highlights. Decihertz observations would:

1. Reveal the formation channels of stellar-mass binaries, complementing groundbased observations with deep multiband observations.

2. Complete our census of the population of BHs by enabling unrivaled measurements of intermediate-mass BHs (IMBHs), which may be the missing step in the evolution of (super)massive BHs.

3. Provide a new laboratory for tests of fundamental physics. 
Decihertz observatories (DOs) have the capability to resolve outstanding questions about the intricate physics of binary stellar evolution, the formation of astrophysical BHs at all scales across cosmic time, and whether extensions to GR or the Standard Model of particle physics are required.

\section{The potential of decihertz observatories}

Decihertz observations would bridge space-based low-frequency detectors and ground-based detectors, giving us access to a wide variety of astrophysical systems:

1. Stellar-mass binaries comprised of compact stellar objects-white dwarfs (WDs), NSs, and stellar-mass BHs. Since BH and NS mergers are observable with ground-based detectors, a DO would allow multiband observations of these populations. WDs are inaccessible to ground-based detectors [27], and so can only be studied with space-based detectors. While the current-generation of ground-based detectors will detect stellar-mass BBHs to redshifts $z \sim 1-$ 2 , next-generation detectors will discover them out to $z \sim 20$, enabling them to chart the evolution of the binary population across the history of the Universe [28]; a DO could match this range, far surpassing LISA. Furthermore, decihertz observations of compact-object mergers would provide valuable forewarning of multimessenger emission associated with merger events. If detected, multimessenger observations reveal details about the equation of state of nuclear density matter [29-33], the production of heavy elements [34-38], and provide a unique laboratory for testing gravity [39-42], as well as potentially identifying the progenitors of Type Ia supernovae [43-45]. Even without finding a counterpart, correlation with galaxy catalogues can provide standard siren cosmological measurements [46-53]. Following their detection by LIGO and Virgo, BHs and NSs are a guaranteed class of GW source [3, 7, 54]. With a large number of observations, we can infer the formation channels for compact-object binaries, and the physics that governs them [28, 55-62]. Eccentricity is a strong indicator of formation mechanism [19, 20, 63-66]; however, residual eccentricity is expected to be small in the regime observable with ground-based detectors [6770] while in some cases, BBHs formed with the highest eccentricities will emit GWs of frequencies too high for LISA [65, 71-76]. Hence DOs could provide unique insights into binary evolution.

2. IMBHs of $\sim 10^{2}-10^{4} M_{\odot}$. IMBHs could be formed via repeated mergers of stars and compact stellar remnants in dense star clusters [77-80]. Using GWs, IMBHs could be observed in a binary with a compact stellar remnant as an intermediate mass-ratio inspiral (IMRI) [81-84], or in a coalescing binary with another IMBH. A DO would enhance the prospects of IMRI detection to tens of events per year, with observations extending out to high redshift. Mergers involving a WD or a NS can lead to tidal disruption events with a bright electromagnetic counterpart [85, 86]. IMBHs binaries could be studied across the entire history of the Universe, charting the properties of this population and constraining the upper end of the pair-instability mass gap [87], while also 
providing a detailed picture of the connection (or lack thereof) between IMBHs and the seeds of massive black holes [88]. The connection between massive BHs and their lower-mass counterparts could be further explored through observations of binaries orbiting massive BHs in galactic centres, or around IMBHs in smaller clusters [76, 89-97]. BBH-IMBH systems are a target for DOground-based multiband observation because they emit both $1-10^{2} \mathrm{~Hz}$ GWs and simultaneously $0.01-1 \mathrm{~Hz}$ GWs.

3. Cosmological sources as part of a stochastic GW background (SGWB). Both this and the other astrophysical sources serve as probes of new physics, enabling tests of deviations from GR and the Standard Model. A first-order phase transition in the early Universe can generate a SGWB [98-103]; a DO would be sensitive to first-order phase transitions occurring at higher temperature, or with a shorter duration, compared to LISA. A DO would be sensitive to a SGWB from a source at $\sim 1 \mathrm{TeV}$ and beyond: $\mathrm{TeV}$-scale phenomena have been consider to resolve with the hierarchy problem or the question of dark matter [104-109], while $100 \mathrm{TeV}$-scale phenomena appear in new solutions to the hierarchy problem such as the relaxion $[110,111]$. Furthermore, SGWB (non-)detection could constrain the properties of cosmic strings [112,113] down to string tensions of $\sim 10^{-19}$, while LISA would reach $\sim 10^{-17}[114]$ and pulsar timing array observations currently constrain tensions to be $\lesssim 10^{-11}[115,116]$.

Decihertz observations provide a unique insight into the physics of each of these sources, and observations would answer questions on diverse topics ranging from the dynamics of globular clusters to the nature of dark matter.

\section{Decihertz mission concepts}

The scientific return of a DO will depend upon its design. There are multiple potential technologies and mission concepts for observing the $0.01-1 \mathrm{~Hz} \mathrm{GW}$ spectrum. The Advanced Laser Interferometer Antenna (ALIA) [117, 118] is a heliocentric mission concept more sensitive than LISA in the $0.1-1 \mathrm{~Hz}$ range. Other heliocentric DO concepts are Taiji $[119,120]$, most sensitive around $0.01 \mathrm{~Hz}$, and TianGo [121], most sensitive in the $0.1-10 \mathrm{~Hz}$ range. TianQin [122] is a Chinese geocentric mission concept. The DECi-hertz Interferometer Gravitational-wave Observatory $(D E C I G O)[123,124]$ is a more ambitious concept with 1000 km Fabry-Perot cavity arms in heliocentric orbit; its precursor $B-D E C I G O$ would be a $100 \mathrm{~km}$ triangular interferometer in a geocentric orbit. The Big Bang Observer $(B B O)$ is a concept consisting of four LISA detectors in heliocentric orbits with combined peak sensitivity over 0.1-1 Hz range [125]. More modest designs are the Geostationary Antenna for Disturbance-Free Laser Interferometry (GADFLI) [126] and geosynchronous Laser Interferometer Space Antenna (gLISA) [127, 128] which are geocentric concepts. The SagnAc interferometer for Gravitational wavE (SAGE) [129, 130] consists of three identical CubeSats in geosynchronous orbit. These concepts are mostly variations on the classic LISA design of a laser interferometer. In addition to laser interferometry, atomic-clock-based [131, 132] and atom interferometer concepts are 
in development; for example, the Mid-band Atomic Gravitational Wave Interferometric Sensor (MAGIS) [133] and the Atomic Experiment for Dark Matter and Gravity Exploration in Space (AEDGE) [134] designs use atom interferometry. The range of technologies available mean that there are multiple possibilities for obtaining the necessary sensitivity in the decihertz range. Two illustrative LISA-like designs, the more ambitious DO-Optimal and the less challenging DO-Conservative, are presented in [26] to assess the potential range of science achievable with DOs. Potential sensitivity of DOs are illustrated in Fig. 1 in comparison to other gravitational-wave observatories.

\section{Summary}

Observing GWs in the decihertz range presents huge opportunities for advancing our understanding of both astrophysics and fundamental physics. The only prospect for decihertz observations is a space-based DO. Realising the rewards of these observations will require development of new detectors beyond LISA. There are many

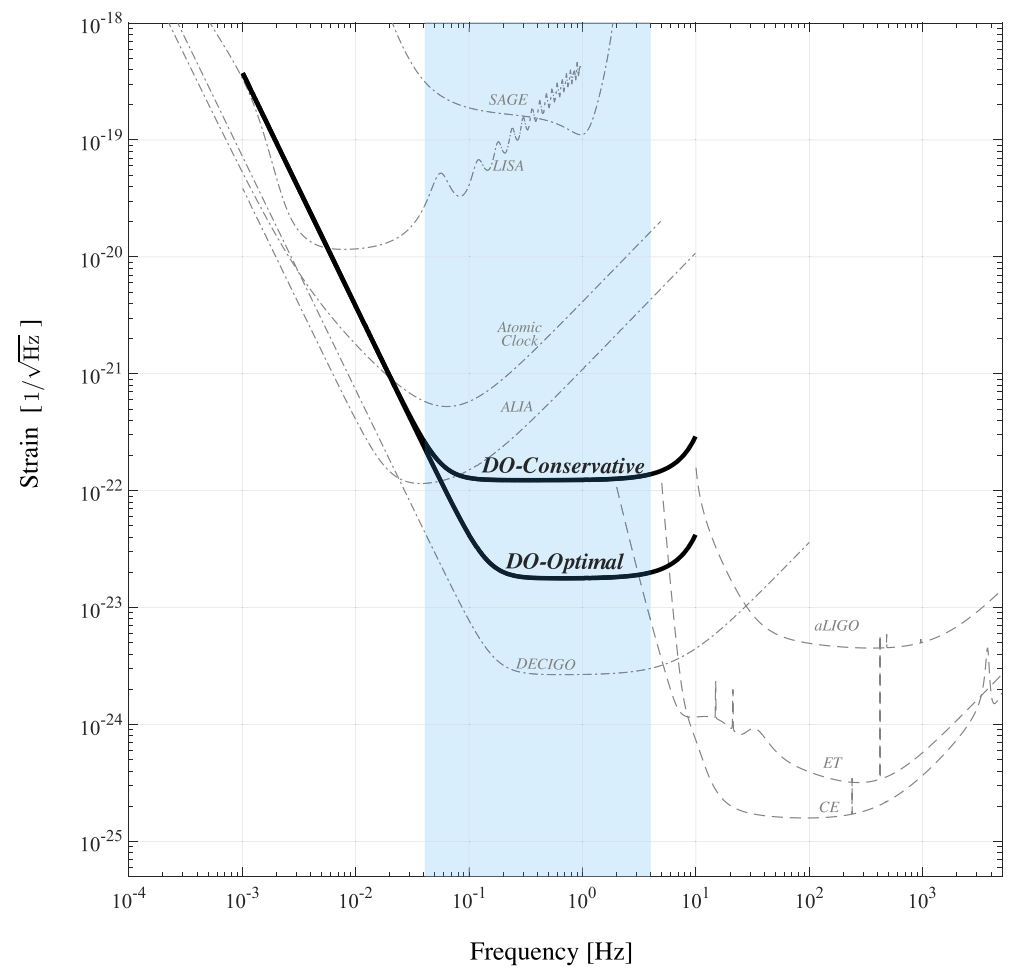

Fig. 1 Concept designs for Decihertz Observatories (DOs) fill the gap between LISA [11] and groundbased detectors like Advanced LIGO (aLIGO) [4], Cosmic Explorer (CE) [8] and the Einstein Telescope (ET) [10]. The example DO concepts $S A G E$ [129, 130], Atomic Clock [26, 131], ALIA [117, 118], DOConservative, DO-Optimal [26, 135] and DECIGO [123, 124] span a diverse set of technologies and illustrate the potential range in sensitivities 
challenges in meeting the requirements of DO concepts; however, there are also many promising technologies that could be developed to meet these goals. A DO mission ready for launch in 2035-2050 is achievable, and the science payoff is worth the experimental effort.

Acknowledgements This summary is derived from a White Paper submitted 4 August 2019 to ESA's Voyage 2050 planning cycle on behalf of the LISA Consortium 2050 Task Force [135]. Further space-based GW observatories considered by the LISA Consortium 2050 Task Force include a microhertz observatory $\mu$ Ares [136]; a more sensitive millihertz observatory, the Advanced Millihertz Gravitational-wave Observatory (AMIGO) [137], and a high angular-resolution observatory consisting of multiple DOs [138].

The authors thanks Pete Bender for insightful comments, and Adam Burrows and David Vartanyan for further suggestions. MAS acknowledges financial support from the Alexander von Humboldt Foundation and the Deutsche Forschungsgemeinschaft (DFG, German Research Foundation) - Project-ID 138713538 - SFB 881 ("The Milky Way System"). CPLB is supported by the CIERA Board of Visitors Research Professorship. PAS acknowledges support from the Ramón y Cajal Programme of the Ministry of Economy, Industry and Competitiveness of Spain, as well as the COST Action GWverse CA16104. This work was supported by the National Key R\&D Program of China (2016YFA0400702) and the National Science Foundation of China (11721303). TB is supported by The Royal Society (grant $\mathrm{URF} \backslash \mathrm{R} 1 \backslash 180009$ ). EB is supported by National Science Foundation (NSF) Grants No. PHY-1912550 and AST-1841358, NASA ATP Grants No. 17-ATP17-0225 and 19-ATP19-0051, NSF-XSEDE Grant No. PHY-090003, and by the Amaldi Research Center, funded by the MIUR program "Dipartimento di Eccellenza" (CUP: B81I18001170001). This work has received funding from the European Union's Horizon 2020 research and innovation programme under the Marie Skłodowska-Curie grant agreement No. 690904. DD acknowledges financial support via the Emmy Noether Research Group funded by the German Research Foundation (DFG) under grant no. DO 1771/1-1 and the Eliteprogramme for Postdocs funded by the Baden-Wurttemberg Stiftung. JME is supported by NASA through the NASA Hubble Fellowship grant HST-HF2-51435.001-A awarded by the Space Telescope Science Institute, which is operated by the Association of Universities for Research in Astronomy, Inc., for NASA, under contract NAS5-26555. MLK acknowledges support from the NSF under grant DGE-0948017 and the Chateaubriand Fellowship from the Office for Science \& Technology of the Embassy of France in the United States. GN is partly supported by the ROMFORSK grant Project No. 302640

'Gravitational Wave Signals From Early Universe Phase Transitions' . IP acknowledges funding by Society in Science, The Branco Weiss Fellowship, administered by the ETH Zurich. AS is supported by the European Union's H2020 ERC Consolidator Grant "Binary massive black hole astrophysics" (grant agreement no. 818691 - B Massive). LS was supported by the National Natural Science Foundation of China (11975027, 11991053, 11721303), the Young Elite Scientists Sponsorship Program by the China Association for Science and Technology (2018QNRC001), and the Max Planck Partner Group Program funded by the Max Planck Society. NW is supported by a Royal Society-Science Foundation Ireland University Research Fellowship (grant UF160093).

Open Access This article is licensed under a Creative Commons Attribution 4.0 International License, which permits use, sharing, adaptation, distribution and reproduction in any medium or format, as long as you give appropriate credit to the original author(s) and the source, provide a link to the Creative Commons licence, and indicate if changes were made. The images or other third party material in this article are included in the article's Creative Commons licence, unless indicated otherwise in a credit line to the material. If material is not included in the article's Creative Commons licence and your intended use is not permitted by statutory regulation or exceeds the permitted use, you will need to obtain permission directly from the copyright holder. To view a copy of this licence, visit http://creativecommons.org/licenses/by/4.0/.

\section{References}

1. Longair, M.: The Cosmic Century. Cambridge University Press, Cambridge (2006). https://doi.org/ 10.1017/CBO9781139878319

2. Sathyaprakash, B.S., Schutz, B.F.: Physics, astrophysics and cosmology with gravitational waves. Living Rev. Rel. 12, 2 (2009). https://doi.org/10.12942/lrr-2009-2 
3. Abbott, B.P., et al.: Observation of gravitational waves from a binary black hole merger. Phys. Rev. Lett. 116(6), 061102 (2016). https://doi.org/10.1103/PhysRevLett.116.061102

4. Aasi, J., et al.: Advanced LIGO. Class. Quant. Grav. 32, 074001 (2015). https://doi.org/10.1088/ 0264-9381/32/7/074001

5. Acernese, F., et al.: Advanced Virgo: A second-generation interferometric gravitational wave detector. Class. Quant. Grav. 32(2), 024001 (2015). https://doi.org/10.1088/0264-9381/32/2/024001

6. Akutsu, T., et al.: KAGRA: 2.5 generation interferometric gravitational wave detector. Nature Astron. 3(1), 35 (2019). https://doi.org/10.1038/s41550-018-0658-y

7. Abbott, B.P., et al.: GWTC-1: A gravitational-wave transient catalog of compact binary mergers observed by LIGO and Virgo during the first and second observing runs. Phys. Rev. x9(3), 031040 (2019). https://doi.org/10.1103/PhysRevX.9.031040

8. Abbott, B.P., et al.: Exploring the sensitivity of next generation gravitational wave detectors. Class. Quant. Grav. 34(4), 044001 (2017). https://doi.org/10.1088/1361-6382/aa51f4

9. Sathyaprakash, B., et al.: Scientific objectives of einstein telescope. Class. Quant. Grav. 29, $124013 \quad$ (2012). https://doi.org/10.1088/0264-9381/29/12/124013, https://doi.org/10.1088/0264-9381/30/7/079501. [Erratum: Class.Quant.Grav.30 079501 (2013)]

10. Hild, S., et al.: Sensitivity studies for third-generation gravitational wave observatories. Class. Quant. Grav. 28, 094013 (2011). https://doi.org/10.1088/0264-9381/28/9/094013

11. Amaro-Seoane, P., et al.: Laser interferometer space antenna. arXiv:1702.00786 (2017)

12. Klein, A., et al.: Science with the space-based interferometer eLISA: Supermassive black hole binaries. Phys. Rev. d93(2), 024003 (2016). https://doi.org/10.1103/PhysRevD.93.024003

13. Babak, S., Gair, J., Sesana, A., Barausse, E., Sopuerta, C.F., Berry, C.P.L., Berti, E., Amaro-Seoane, P., Petiteau, A., Klein, A.: Science with the space-based interferometer LISA. V: Extreme mass-ratio inspirals. Phys. Rev. d95(10), 103012 (2017). https://doi.org/10.1103/PhysRevD.95.103012

14. Berry, C.P.L., Hughes, S.A., Sopuerta, C.F., Chua, A.J.K., Heffernan, A., Holley-Bockelmann, K., Mihaylov, D.P., Miller, M.C., Sesana, A.: The unique potential of extreme mass-ratio inspirals for gravitational-wave astronomy. Bull. Am. Astron. Soc. 51(3), 42 (2019)

15. Sesana, A.: Prospects for Multiband gravitational-wave astronomy after GW150914. Phys. Rev. Lett. 116(23), 231102 (2016). https://doi.org/10.1103/PhysRevLett.116.231102

16. Vitale, S.: Multiband gravitational-wave astronomy: Parameter estimation and tests of general relativity with space- and ground-based detectors. Phys. Rev. Lett. 117(5), 051102 (2016). https://doi.org/10.1103/PhysRevLett.117.051102

17. Jani, K., Shoemaker, D., Cutler, C.: Detectability of intermediate-mass black holes in Multiband gravitational wave astronomy. Nature Astron. 4(3), 260 (2019). https://doi.org/10.1038/s41550019-0932-7

18. Liu, C., Shao, L., Zhao, J., Gao, Y.: Multiband observation of LIGO/Virgo binary black hole mergers in the gravitational-wave transient catalog GWTC-1. Mon. Not. Roy. Astron. Soc. 496, 182 (2020). https://doi.org/10.1093/mnras/staa1512

19. Breivik, K., Rodriguez, C.L., Larson, S.L., Kalogera, V., Rasio, F.A.: Distinguishing between formation channels for binary black holes with LISA. Astrophys. J. 830(1), L18 (2016). https://doi.org/10.3847/2041-8205/830/1/L18

20. Nishizawa, A., Berti, E., Klein, A., Sesana, A.: eLISA eccentricity measurements as tracers of binary black hole formation. Phys. Rev. d94(6), 064020 (2016). https://doi.org/10.1103/PhysRevD. 94.064020

21. Toubiana, A., Marsat, S., Babak, S., Barausse, E., Baker, J.: Tests of general relativity with stellar-mass black hole binaries observed by LISA. Phys. Rev. d101(10), 104038 (2020). https://doi.org/10.1103/PhysRevD.101.104038

22. Manchester, R.N.: The international pulsar timing array. Quant, Class. Grav. 30, 224010 (2013). https://doi.org/10.1088/0264-9381/30/22/224010

23. Mingarelli, C.M.F., Lazio, T.J.W., Sesana, A., Greene, J.E., Ellis, J.A., Ma, C.P., Croft, S., BurkeSpolaor, S., Taylor, S.R.: The local Nanohertz gravitational-wave landscape from supermassive black hole binaries. Nature Astron. 1(12), 886 (2017). https://doi.org/10.1038/s41550-017-0299-6

24. Pitkin, M., Clark, J., Hendry, M.A., Heng, I.S., Messenger, C., Toher, J., Woan, G., Phys, J.: Is there potential complementarity between LISA and pulsar timing? Conf. Ser. 122, 012004 (2008). https://doi.org/10.1088/1742-6596/122/1/012004

25. Colpi, M., et al.: The gravitational wave view of massive black holes. Bull. Am. Astron. Soc. 51(3), 432 (2019) 
26. Sedda, M.A., et al.: The missing link in gravitational-wave astronomy: Discoveries waiting in the decihertz range. Class. Quant. Grav. 37(21), 215011 (2020). https://doi.org/10.1088/1361$6382 / \mathrm{abb} 5 \mathrm{c} 1$

27. Littenberg, T.B., Breivik, K., Brown, W.R., Eracleous, M., Hermes, J.J., Holley-Bockelmann, K., Kremer, K., Kupfer, T., Larson, S.L.: Gravitational wave survey of galactic ultra compact binaries. Bull. Am. Astron. Soc. 51(3), 34 (2019)

28. Kalogera, V., et al.: Deeper, Wider, Sharper: Next-generation ground-based gravitational-wave observations of binary black holes. Bull. Am. Astron. Soc. 51(3), 242 (2019)

29. Abbott, B.P., et al.: GW170817: Measurements of neutron star radii and equation of state. Phys. Rev. Lett. 121(16), 161101 (2018). https://doi.org/10.1103/PhysRevLett.121.161101

30. Montana, G., Tolos, L., Hanauske, M., Rezzolla, L.: Constraining twin stars with GW170817. Phys. Rev. d99(10), 103009 (2019). https://doi.org/10.1103/PhysRevD.99.103009

31. Most, E.R., Weih, L.R., Rezzolla, L., Schaffner-Bielich, J.: New constraints on radii and tidal deformabilities of neutron stars from GW170817. Phys. Rev. Lett. 120(26), 261103 (2018). https://doi.org/10.1103/PhysRevLett.120.261103

32. Coughlin, M.W., Dietrich, T., Margalit, B., Metzger, B.D.: Multimessenger Bayesian parameter inference of a binary neutron star merger. Mon. Not. Roy. Astron. Soc. 489(1), L91 (2019). https://doi.org/10.1093/mnrasl/slz133

33. Margalit, B., Metzger, B.D.: The multi-messenger matrix: The future of neutron star merger constraints on the nuclear equation of state. Astrophys. J. 880(1), L15 (2019). https://doi.org/10.3847/ 2041-8213/ab2ae2

34. Abbott, B.P., et al.: Estimating the contribution of dynamical ejecta in the Kilonova associated with GW170817. Astrophys. J. 850(2), L39 (2017). https://doi.org/10.3847/2041-8213/aa9478

35. Chornock, R., et al.: The electromagnetic counterpart of the binary neutron star merger LIGO/Virgo GW170817. IV. Detection of Near-infrared Signatures of r-process Nucleosynthesis with GeminiSouth. Astrophys. J. 848(2), L19 (2017). https://doi.org/10.3847/2041-8213/aa905c

36. Tanvir, N.R., et al.: The emergence of a Lanthanide-Rich Kilonova following the merger of two neutron stars. Astrophys. J. 848(2), L27 (2017). https://doi.org/10.3847/2041-8213/aa90b6

37. Wanajo, S.: Physical conditions for the r-process I. radioactive energy sources of kilonovae. Astrophys. J. 868(1), 65 (2018). https://doi.org/10.3847/1538-4357/aae0f2

38. Siegel, D.M., Barnes, J., Metzger, B.D.: The neutron star merger GW170817 points to collapsars as the main r-process source. Nature 569(7755), 241 (2019). https://doi.org/10.1038/s41586019-1136-0

39. Abbott, B.P., et al:: Gravitational waves and gamma-rays from a binary neutron star merger: GW170817 and GRB 170817A. Astrophys. J. 848(2), L13 (2017). https://doi.org/10.3847/20418213/aa920c

40. Abbott, B.P., et al.: Tests of general relativity with GW170817. Phys. Rev. Lett. 123(1), 011102 (2019). https://doi.org/10.1103/PhysRevLett.123.011102

41. Belgacem, E., Dirian, Y., Foffa, S., Maggiore, M.: Modified gravitational-wave propagation and standard sirens. Phys. Rev. d98(2), 023510 (2018). https://doi.org/10.1103/PhysRevD.98.023510

42. Belgacem, E., et al.: Testing modified gravity at cosmological distances with LISA standard sirens. JCAP 1907(07), 024 (2019). https://doi.org/10.1088/1475-7516/2019/07/024

43. Hillebrandt, W., Kromer, M., Röpke, F.K., Ruiter, A.J.: Towards an understanding of Type Ia supernovae from a synthesis of theory and observations. Front. Phys. (Beijing) 8, 116 (2013). https://doi.org/10.1007/s11467-013-0303-2

44. Maoz, D., Mannucci, F., Nelemans, G.: Observational clues to the progenitors of Type-Ia supernovae. Ann. Rev. Astron. Astrophys. 52, 107 (2014). https://doi.org/10.1146/annurev-astro-082812-141031

45. Mandel, I., Sesana, A., Vecchio, A.: The astrophysical science case for a decihertz gravitational-wave detector. Class. Quant. Grav. 35(5), 054004 (2018). https://doi.org/10.1088/1361-6382/aaa7e0

46. Schutz, B.F.: Determining the hubble constant from gravitational wave observations. Nature 323, 310 (1986). https://doi.org/10.1038/323310a0

47. MacLeod, C.L., Hogan, C.J.: Precision of Hubble constant derived using black hole binary absolute distances and statistical redshift information. Phys. Rev. D77, 043512 (2008). https://doi.org/10.1103/PhysRevD.77.043512

48. Chen, H.Y., Fishbach, M., Holz, D.E.: A two per cent Hubble constant measurement from standard sirens within five years. Nature 562(7728), 545 (2018). https://doi.org/10.1038/s41586-018-0606-0 
49. Abbott, B.P., et al.: A gravitational-wave measurement of the Hubble constant following the second observing run of Advanced LIGO and Virgo. Astrophys. J. 909(2), 218 (2021). https://doi.org/10.3847/ $1538-4357 / \mathrm{abdcb} 7$

50. Kyutoku, K., Seto, N.: Gravitational-wave cosmography with LISA and the Hubble tension. Phys. Rev. d95(8), 083525 (2017). https://doi.org/10.1103/PhysRevD.95.083525

51. Del Pozzo, W., Sesana, A., Klein, A.: Stellar binary black holes in the LISA band: a new class of standard sirens. Mon. Not. Roy. Astron. Soc. 475(3), 3485 (2018). https://doi.org/10.1093/mnras/sty057

52. Cutler, C., Holz, D.E.: Ultra-high precision cosmology from gravitational waves. Phys. Rev. D80, 104009 (2009). https://doi.org/10.1103/PhysRevD.80.104009

53. Nishizawa, A., Taruya, A., Saito, S.: Tracing the redshift evolution of Hubble parameter with gravitational-wave standard sirens. Phys. Rev. D83, 084045 (2011). https://doi.org/10.1103/ PhysRevD.83.084045

54. Abbott, R., et al.: GWTC-2: Compact Binary Coalescences observed by LIGO and Virgo during the first half of the third observing run. arXiv:2010.14527 (2020)

55. Mandel, I., O'Shaughnessy, R.: Compact binary Coalescences in the band of ground-based gravitational-wave detectors. Class. Quant. Grav. 27, 114007 (2010). https://doi.org/10.1088/02649381/27/11/114007

56. Stevenson, S., Berry, C.P.L., Mandel, I.: Hierarchical analysis of gravitational-wave measurements of binary black hole spinorbit misalignments. Mon. Not. Roy. Astron. Soc. 471(3), 2801 (2017). https://doi.org/10.1093/mnras/stx1764

57. Talbot, C., Thrane, E.: Determining the population properties of spinning black holes. Phys. Rev. d96(2), 023012 (2017). https://doi.org/10.1103/PhysRevD.96.023012

58. Zevin, M., Pankow, C., Rodriguez, C.L., Sampson, L., Chase, E., Kalogera, V., Rasio, F.A.: Constraining formation models of binary black holes with gravitational-wave observations. Astrophys. J. 846(1), 82 (2017). https://doi.org/10.3847/1538-4357/aa8408

59. Barrett, J.W., Gaebel, S.M., Neijssel, C.J., Vigna-Gómez, A., Stevenson, S., Berry, C.P.L., Farr, W.M., Mandel, I.: Accuracy of inference on the physics of binary evolution from gravitationalwave observations. Mon. Not. Roy. Astron. Soc. 477(4), 4685 (2018). https://doi.org/10.1093/ mnras/sty908

60. Arca Sedda, M., Benacquista, M.: Using final black hole spins and masses to infer the formation history of the observed population of gravitational wave sources. Mon. Not. Roy. Astron. Soc. 482(3), 2991 (2019). https://doi.org/10.1093/mnras/sty2764

61. Arca Sedda, M., Mapelli, M., Spera, M., Benacquista, M., Giacobbo, N.: Fingerprints of binary black hole formation channels encoded in the mass and spin of merger remnants. Astrophys. J. 894(2), 133 (2020). https://doi.org/10.3847/1538-4357/ab88b2

62. Farmer, R., Renzo, M., de Mink, S., Fishbach, M., Justham, S.: Constraints from gravitational wave detections of binary black hole mergers on the ${ }^{12} \mathrm{C}(\alpha, \gamma){ }^{16} \mathrm{O}$ rate. Astrophys. J. Lett. 902(2), L36 (2020). https://doi.org/10.3847/2041-8213/abbadd

63. Nishizawa, A., Sesana, A., Berti, E., Klein, A.: Constraining stellar binary black hole formation scenarios with eLISA eccentricity measurements. Mon. Not. Roy. Astron. Soc. 465(4), 4375 (2017). https://doi.org/10.1093/mnras/stw2993

64. Canuel, B., et al.: Exploring gravity with the MIGA large scale atom interferometer. Sci. Rep. 8(1), 14064 (2018). https://doi.org/10.1038/s41598-018-32165-z

65. Kremer, K., Chatterjee, S., Breivik, K., Rodriguez, C.L., Larson, S.L., Rasio, F.A.: LISA sources in Milky Way globular clusters. Phys. Rev. Lett. 120(19), 191103 (2018). https://doi.org/10.1103/PhysRevLett.120.191103

66. Randall, L., Xianyu, Z.Z.: Eccentricity without measuring eccentricity: Discriminating among stellar mass black hole binary formation channels. arXiv:1907.02283 (2019)

67. Peters, P.C.: Gravitational radiation and the motion of two point masses. Phys. Rev. 136, B1224 (1964). https://doi.org/10.1103/PhysRev.136.B1224

68. Abbott, B.P., et al.: Astrophysical implications of the binary black-hole merger GW150914. Astrophys. J. 818(2), L22 (2016). https://doi.org/10.3847/2041-8205/818/2/L22

69. Samsing, J., Ramirez-Ruiz, E.: On the assembly rate of highly eccentric binary black hole mergers. Astrophys. J. 840(2), L14 (2017). https://doi.org/10.3847/2041-8213/aa6f0b

70. Rodriguez, C.L., Amaro-Seoane, P., Chatterjee, S., Kremer, K., Rasio, F.A., Samsing, J., Ye, C.S., Zevin, M.: Post-Newtonian dynamics in dense star clusters: Formation, Masses, and 
merger rates of highly-eccentric black hole binaries. Phys. Rev. d98(12), 123005 (2018). https://doi.org/10.1103/PhysRevD.98.123005

71. Randall, L., Xianyu, Z.Z.: A direct probe of mass density near inspiraling binary black holes. Astrophys. J. 878(2), 75 (2019). https://doi.org/10.3847/1538-4357/ab20c6

72. D’Orazio, D.J., Samsing, J.: Black hole mergers from globular clusters observable by LISA II: Resolved eccentric sources and the gravitational wave background. Mon. Not. Roy. Astron. Soc. 481(4), 4775 (2018). https://doi.org/10.1093/mnras/sty2568

73. Arca-Sedda, M., Li, G., Kocsis, B.: Ordering the chaos: stellar black hole mergers from nonhierarchical triples. arXiv:1805.06458 (2018)

74. Kremer, K., et al.: Post-Newtonian dynamics in dense star clusters: Binary black holes in the LISA Band. Phys. Rev. d99(6), 063003 (2019). https://doi.org/10.1103/PhysRevD.99.063003

75. Zevin, M., Samsing, J., Rodriguez, C., Haster, C.J., Ramirez-Ruiz, E.: Eccentric black hole mergers in dense star clusters: The Role of Binary Encounters. Astrophys. J. 871(1), 91 (2019). https://doi.org/10.3847/1538-4357/aaf6ec

76. Chen, X., Amaro-Seoane, P.: Revealing the formation of stellar-mass black hole binaries: The need for deci-Hertz gravitational wave observatories. Astrophys. J. 842(1), L2 (2017). https://doi.org/10.3847/2041-8213/aa74ce

77. Portegies Zwart, S.F., McMillan, S.: Black hole mergers in the universe. Astrophys. J. 528, L17 (2000). https://doi.org/10.1086/312422

78. Giersz, M., Leigh, N., Hypki, A., Lützgendorf, N., Askar, A.: MOCCA code for star cluster simulations - IV. A new scenario for intermediate mass black hole formation in globular clusters. Mon. Not. Roy. Astron. Soc. 454(3), 3150 (2015). https://doi.org/10.1093/mnras/stv2162

79. Arca Sedda, M., Askar, A., Giersz, M.: MOCCA-SURVEY Database I. Intermediate mass black holes in Milky Way globular clusters and their connection to supermassive black holes. arXiv:1905.00902 (2019)

80. Abbott, R., et al.: Properties and Astrophysical Implications of the $150 \quad \mathbf{M}_{\odot}$ Binary Black Hole Merger GW190521. Astrophys. J. Lett. 900(1), L13 (2020). https://doi.org/10.3847/2041-8213/aba493

81. Amaro-Seoane, P., Gair, J.R., Freitag, M., Coleman Miller, M., Mandel, I., Cutler, C.J., Babak, S.: Astrophysics, detection and science applications of intermediate- and extreme mass-ratio inspirals. Class. Quant. Grav. 24, R113 (2007). https://doi.org/10.1088/0264-9381/24/17/R01

82. Brown, D.A., Fang, H., Gair, J.R., Li, C., Lovelace, G., Mandel, I., Thorne, K.S.: Prospects for detection of gravitational waves from intermediate-mass-ratio inspirals. Phys. Rev. Lett. 99, 201102 (2007). https://doi.org/10.1103/PhysRevLett.99.201102

83. Rodriguez, C.L., Mandel, I., Gair, J.R.: Verifying the no-hair property of massive compact objects with intermediate-mass-ratio inspirals in advanced gravitational-wave detectors. Phys. Rev. D85, 062002 (2012). https://doi.org/10.1103/PhysRevD.85.062002

84. Haster, C.J., Wang, Z., Berry, C.P.L., Stevenson, S., Veitch, J., Mandel, I.: Inference on gravitational waves from coalescences of stellar-mass compact objects and intermediate-mass black holes. Mon. Not. Roy. Astron. Soc. 457(4), 4499 (2016). https://doi.org/10.1093/mnras/stw233

85. Chen, J.H., Shen, R.F.: Tidal disruption of a main-sequence star by an intermediate-mass black hole: A bright decade. Astrophys. J. 867(1), 20 (2018). https://doi.org/10.3847/1538-4357/aadfda

86. Eracleous, M., Gezari, S., Sesana, A., Bogdanovic, T., MacLeod, M., Roth, N., Dai, L.: An arena for multi-messenger astrophysics: Inspiral and tidal disruption of white dwarfs by massive black holes. Bull. Am. Astron. Soc. 51(3), 10 (2019)

87. Ezquiaga, J.M., Holz, D.E.: Jumping the gap: searching for LIGO's biggest black holes. arXiv:2006.02211. Astrophys. J. Lett. 909(2), L23 (2020)

88. Volonteri, M., Natarajan, P.: Journey to the $M_{\mathrm{B} H}-\sigma$ relation: the fate of low mass black holes in the Universe. Mon. Not. Roy. Astron. Soc. 400, 1911 (2009). https://doi.org/10.1111/j.1365-2966. 2009.15577.x

89. McKernan, B., Ford, K.E.S., Kocsis, B., Lyra, W., Winter, L.M.: Intermediate-mass black holes in AGN discs II. Model predictions and observational constraints. Mon. Not. Roy. Astron. Soc. 441(1), 900 (2014). https://doi.org/10.1093/mnras/stu553

90. Bartos, I., Kocsis, B., Haiman, Z., Márka, S.: Rapid and bright stellar-mass binary black hole mergers in active galactic nuclei. Astrophys. J. 835(2), 165 (2017). https://doi.org/https://doi.org/10.3847/ $1538-4357 / 835 / 2 / 165$ 
91. Stone, N.C., Metzger, B.D., Haiman, Z.: Assisted inspirals of stellar mass black holes embedded in AGN discs: solving the final au problem. Mon. Not. Roy. Astron. Soc. 464(1), 946 (2017). https://doi.org/10.1093/mnras/stw2260

92. McKernan, B., et al.: Constraining stellar-mass black hole mergers in AGN Disks detectable with LIGO. Astrophys. J. 866(1), 66 (2018). https://doi.org/10.3847/1538-4357/aadae5

93. Chen, X., Li, S., Cao, Z.: Massaredshift degeneracy for the gravitational-wave sources in the vicinity of supermassive black holes. Mon. Not. Roy. Astron. Soc. 485(1), L141 (2019). https://doi.org/10.1093/mnrasl/slz046

94. Gondán, L., Kocsis, B., Raffai, P., Frei, Z.: Eccentric black hole gravitational-wave capture sources in galactic nuclei: Distribution of binary parameters. Astrophys. J. 860(1), 5 (2018). https://doi.org/10.3847/1538-4357/aabfee

95. Secunda, A., Bellovary, J., Mac Low, M.M., Saavik Ford, K.E., McKernan, B., Leigh, N., Lyra, W., Sándor, Z.: Orbital migration of interacting stellar mass black holes in disks around supermassive black holes. Astrophys. J. 878(2), 85 (2019). https://doi.org/10.3847/1538-4357/ab20ca

96. Rasskazov, A., Kocsis, B.: The rate of stellar mass black hole scattering in galactic nuclei. Astrophys. J. 881, 20 (2019). https://doi.org/10.3847/1538-4357/ab2c74

97. Yang, Y., Bartos, I., Haiman, Z., Kocsis, B., Marka, Z., Stone, N.C., Marka, S.: AGN disks harden the mass distribution of stellar-mass binary black hole mergers. Astrophys. J. 876(2), 122 (2019). https://doi.org/10.3847/1538-4357/ab16e3

98. Kosowsky, A., Turner, M.S.: Gravitational radiation from colliding vacuum bubbles: Envelope approximation to many bubble collisions. Phys. Rev. D47, 4372 (1993). https://doi.org/10.1103/ PhysRevD.47.4372

99. Kamionkowski, M., Kosowsky, A., Turner, M.S.: Gravitational radiation from first order phase transitions. Phys. Rev. D49, 2837 (1994). https://doi.org/10.1103/PhysRevD.49.2837

100. Gogoberidze, G., Kahniashvili, T., Kosowsky, A.: The spectrum of gravitational radiation from primordial turbulence. Phys. Rev. 083002, D76 (2007). https://doi.org/10.1103/PhysRevD.76.083002

101. Caprini, C., Durrer, R., Servant, G.: The stochastic gravitational wave background from turbulence and magnetic fields generated by a first-order phase transition. JCAP 0912, 024 (2009). https://doi.org/10.1088/1475-7516/2009/12/024

102. Hindmarsh, M., Huber, S.J., Rummukainen, K., Weir, D.J.: Gravitational waves from the sound of a first order phase transition. Phys. Rev. Lett. 112, 041301 (2014). https://doi.org/10.1103/PhysRevLett.112.041301

103. Hindmarsh, M., Huber, S.J., Rummukainen, K., Weir, D.J.: Numerical simulations of acoustically generated gravitational waves at a first order phase transition. Phys. Rev. d92(12), 123009 (2015). https://doi.org/10.1103/PhysRevD.92.123009

104. Randall, L., Servant, G.: Gravitational waves from warped spacetime. JHEP 05, 054 (2007). https://doi.org/10.1088/1126-6708/2007/05/054

105. Nardini, G., Quiros, M., Wulzer, A.: A confining strong first-order electroweak phase transition. JHEP 09, 077 (2007). https://doi.org/10.1088/1126-6708/2007/09/077

106. Konstandin, T., Nardini, G., Quiros, M.: Gravitational backreaction effects on the holographic phase transition. Phys. Rev. D82, 083513 (2010). https://doi.org/10.1103/PhysRevD.82.083513

107. Konstandin, T., Servant, G.: Cosmological consequences of nearly conformal dynamics at the TeV scale. JCAP 1112, 009 (2011). https://doi.org/10.1088/1475-7516/2011/12/009

108. Bruggisser, S., Von Harling, B., Matsedonskyi, O., Servant, G.: Baryon asymmetry from a composite Higgs boson. Phys. Rev. Lett. 121(13), 131801 (2018). https://doi.org/10.1103/PhysRevLett. 121.131801

109. Megías, E., Nardini, G., Quirós, M.: Cosmological phase transitions in warped space: Gravitational Waves and Collider Signatures. JHEP 09, 095 (2018). https://doi.org/10.1007/JHEP09(2018)095

110. Arkani-Hamed, N., Han, T., Mangano, M., Wang, L.T.: Physics opportunities of a $100 \mathrm{TeV}$ protonaproton collider. Phys. Rept. 652, 1 (2016). https://doi.org/10.1016/j.physrep.2016.07.004

111. Graham, P.W., Kaplan, D.E., Mardon, J., Rajendran, S., Terrano, W.A.: Dark matter direct detection with accelerometers. Phys. Rev. d93(7), 075029 (2016). https://doi.org/10.1103/PhysRevD. 93.075029

112. Vachaspati, T., Vilenkin, A.: Gravitational radiation from cosmic strings. Phys. Rev. D31, 3052 (1985). https://doi.org/10.1103/PhysRevD.31.3052

113. Blanco-Pillado, J.J., Olum, K.D., Shlaer, B.: The number of cosmic string loops. Phys. Rev. d89(2), 023512 (2014). https://doi.org/10.1103/PhysRevD.89.023512 
114. Auclair, P., et al.: Probing the gravitational wave background from cosmic strings with LISA. JCAP 2004, 034 (2020). https://doi.org/10.1088/1475-7516/2020/04/034

115. Sanidas, S.A., Battye, R.A., Stappers, B.W.: Constraints on cosmic string tension imposed by the limit on the stochastic gravitational wave background from the European Pulsar Timing Array. Phys. Rev. D85, 122003 (2012). https://doi.org/10.1103/PhysRevD.85.122003

116. Blanco-Pillado, J.J., Olum, K.D., Siemens, X.: New limits on cosmic strings from gravitational wave observation. Phys. Lett. B778, 392 (2018). https://doi.org/10.1016/j.physletb.2018.01.050

117. Bender, P.L., Begelman, M.C., Gair, J.R.: Possible LISA follow-on mission scientific objectives. Class. Quant. Grav. 30, 165017 (2013). https://doi.org/10.1088/0264-9381/30/16/165017

118. Mueller, G., Baker, J., et al.: Space based gravitational wave astronomy beyond LISA. Bull. Am. Astron. Soc. 51(7), 243 (2019)

119. Hu, W.R., Wu, Y.L.: The Taiji Program in Space for gravitational wave physics and the nature of gravity. Natl. Sci. Rev. 4(5), 685 (2017). https://doi.org/10.1093/nsr/nwx116

120. Ruan, W.H., Guo, Z.K., Cai, R.G., Zhang, Y.Z.: Taiji Program: Gravitational-wave sources. Int. J. Mod. Phys. a35(17), 2050075 (2020). https://doi.org/10.1142/S0217751X2050075X

121. Kuns, K.A., Yu, H., Chen, Y., Adhikari, R.X.: Astrophysics and cosmology with a decihertz gravitational-wave detector: TianGO. Phys. Rev. d102(4), 043001 (2020). https://doi.org/10.1103/ PhysRevD.102.043001

122. Luo, J., et al.: TianQin: A space-borne gravitational wave detector. Class. Quant. Grav. 33(3), 035010 (2016). https://doi.org/10.1088/0264-9381/33/3/035010

123. Sato, S., et al.: The status of DECIGO. J. Phys. Conf. Ser. 840(1), 012010 (2017). https://doi.org/ 10.1088/1742-6596/840/1/012010

124. Kawamura, S., et al.: Current status of space gravitational wave antenna DECIGO and B-DECIGO. arXiv:2006.13545 (2020)

125. Crowder, J., Cornish, N.J.: Beyond LISA: Exploring future gravitational wave missions. Phys. Rev. D72, 083005 (2005). https://doi.org/10.1103/PhysRevD.72.083005

126. McWilliams, S.T.: Geostationary antenna for disturbance-free laser interferometry (GADFLI). arXiv:1111.3708 (2011)

127. Tinto, M., de Araujo, J.C.N., Aguiar, O.D., da Silva Alves, M.E.: A geostationary gravitational wave interferometer (GEOGRAWI). arXiv:1111.2576 (2011)

128. Tinto, M., DeBra, D., Buchman, S., Tilley, S.: gLISA: geosynchronous Laser Interferometer Space Antenna concepts with off-the-shelf satellites. Rev. Sci. Instrum. 86, 014501 (2015). https://doi.org/10.1063/1.4904862

129. Lacour, S., et al.: SAGE: finding IMBH in the black hole desert. Class. Quant. Grav. 36(19), 195005 (2019). https://doi.org/10.1088/1361-6382/ab3583

130. Tino, G.M., et al.: SAGE: A proposal for a space atomic gravity explorer. Eur. Phys. J. D73(11), 228 (2019). https://doi.org/10.1140/epjd/e2019-100324-6

131. Kolkowitz, S., Pikovski, I., Langellier, N., Lukin, M.D., Walsworth, R.L., Ye, J.: Gravitational wave detection with optical lattice atomic clocks. Phys. Rev. d94(12), 124043 (2016). https://doi.org/10.1103/PhysRevD.94.124043

132. Su, J., Wang, Q., Wang, Q., Jetzer, P.: Low-frequency gravitational wave detection via double optical clocks in space. Class. Quant. Grav. 35(8), 085010 (2018). https://doi.org/10.1088/ 1361-6382/aaebd2, https://doi.org/10.1088/1361-6382/aab2eb. [Erratum: Class. Quant. Grav.35,no. 24,249501(2018)]

133. Graham, P.W., Hogan, J.M., Kasevich, M.A., Rajendran, S., Romani, R.W.: Mid-band gravitational wave detection with precision atomic sensors. arXiv:1711.02225 (2017)

134. El-Neaj, Y.A., et al.: AEDGE: atomic experiment for dark matter and gravity exploration in space. EPJ Quant. Technol. 7, 6 (2020). https://doi.org/10.1140/epjqt/s40507-020-0080-0

135. Arca Sedda, M., et al.: The Missing Link in Gravitational-Wave Astronomy: Discoveries waiting in the decihertz range. arXiv:1908.11375v1 (2019)

136. Sesana, A., et al.: Unveiling the gravitational universe at $\mu$-Hz Frequencies. arXiv:1908.11391 (2019)

137. Baibhav, V., et al.: Probing the nature of black holes: Deep in the $\mathrm{mHz}$ gravitational-wave sky. arXiv:1908.11390 (2019)

138. Baker, J., et al.: High angular resolution gravitational wave astronomy. arXiv:1908.11410 (2019)

Publisher's note Springer Nature remains neutral with regard to jurisdictional claims in published maps and institutional affiliations. 


\section{Affiliations}

Manuel Arca Sedda ${ }^{1} \cdot$ Christopher P. L. Berry ${ }^{2,3}$ (D) . Karan Jani ${ }^{4}$. Pau Amaro-Seoane $e^{5,6,7,8}$. Pierre Auclair ${ }^{9}$. Jonathon Baird ${ }^{10}$. Tessa Baker ${ }^{11}$. Emanuele Berti $^{12}$. Katelyn Breivik ${ }^{13}$. Chiara Caprini ${ }^{9}$ - Xian Chen ${ }^{6,14}$. Daniela Doneva ${ }^{15}$. Jose M. Ezquiaga ${ }^{16}$. K. E. Saavik Ford ${ }^{17,18}$. Michael L. Katz ${ }^{2}$. Shimon Kolkowitz ${ }^{19}$ - Barry McKernan ${ }^{17,18}$. Guido Mueller ${ }^{20}$. Germano Nardini $^{21}$. Igor Pikovski22,23 . Surjeet Rajendran ${ }^{12}$.

Alberto Sesana ${ }^{24}$. Lijing Shao ${ }^{6,25}$ • Nicola Tamanini ${ }^{26}$. Niels Warburton ${ }^{27}$. Helvi Witek ${ }^{28} \cdot$ Kaze Wong ${ }^{12} \cdot$ Michael Zevin $^{2}$ 
1 Astronomisches Rechen-Institut, Zentrüm für Astronomie, Universität Heidelberg, Mönchofstr. 1214, Heidelberg, Germany

2 Center for Interdisciplinary Exploration and Research in Astrophysics (CIERA), Department of Physics and Astronomy, Northwestern University, 2145 Sheridan Road, Evanston, IL, 60208, USA

3 SUPA, School of Physics and Astronomy, University of Glasgow, Glasgow, G12 8QQ, UK

4 Department of Physics and Astronomy, Vanderbilt University, Nashville, TN, 37212, USA

5 Universitat Politècnica de València, IGIC, Valencia, Spain

6 Kavli Institute for Astronomy and Astrophysics, Peking University, Beijing, 100871, China

7 Institute of Applied Mathematics, Academy of Mathematics and Systems Science, CAS, Beijing, 100190, China

8 Zentrum für Astronomie und Astrophysik, TU Berlin, Hardenbergstraße 36, 10623, Berlin, Germany

9 Laboratoire Astroparticule et Cosmologie, CNRS UMR 7164, Université Paris-Diderot, 10 rue Alice Domon et Léonie Duquet, 75013, Paris, France

10 High Energy Physics Group, Physics Department, Imperial College London, Blackett Laboratory, Prince Consort Road, London, SW7 2BW, UK

11 School of Physics and Astronomy, Queen Mary University of London, Mile End Road, London, E1 4NS, UK

12 Department of Physics and Astronomy, Johns Hopkins University, 3400 N. Charles Street, Baltimore, MD 21218, USA

13 Canadian Institute for Theoretical Astrophysics, University of Toronto, 60 St. George Street, Toronto, Ontario, M5S 1A7, Canada

14 Astronomy Department, School of Physics, Peking University, Beijing, 100871, China

15 Theoretical Astrophysics, Eberhard Karls University of Tübingen, Tübingen, 72076, Germany

16 Kavli Institute for Cosmological Physics, Enrico Fermi Institute, The University of Chicago, Chicago, IL, 60637, USA

17 City University of New York-BMCC, 199 Chambers St, New York, NY, 10007, USA

18 Department of Astrophysics, American Museum of Natural History, New York, NY, 10028, USA

19 Department of Physics, University of Wisconsin - Madison, Madison, WI, 53706, USA

20 Department of Physics, University of Florida, PO Box 118440, Gainesville, Florida, 32611, USA

21 Faculty of Science and Technology, University of Stavanger, 4036, Stavanger, Norway

22 Department of Physics, Stevens Institute of Technology, Hoboken, NJ, 07030, USA

23 Department of Physics, Stockholm University, SE-10691, Stockholm, Sweden

24 Università di Milano Bicocca, Dipartimento di Fisica G. Occhialini, Piazza della Scienza 3, I-20126, Milano, Italy

25 National Astronomical Observatories, Chinese Academy of Sciences, Beijing, 100012, China

26 Max-Planck-Institut für Gravitationsphysik (Albert-Einstein-Institut), Am Mühlenberg 1, 14476, Potsdam-Golm, Germany

27 School of Mathematics and Statistics, University College Dublin, Belfield, Dublin 4, Ireland

28 Department of Physics, King's College London, Strand, London, WC2R 2LS, UK 